Research Article

\title{
Preparation of InSe Thin Films by Thermal Evaporation Method and Their Characterization: Structural, Optical, and Thermoelectrical Properties
}

\author{
Sarita Boolchandani $(\mathbb{D}$, Subodh Srivastava, and Y. K. Vijay \\ Vivekananda Global University, Jaipur, India \\ Correspondence should be addressed to Sarita Boolchandani; saritaboolchandani@gmail.com
}

Received 16 May 2017; Revised 10 November 2017; Accepted 8 January 2018; Published 1 February 2018

Academic Editor: Valery Khabashesku

Copyright (c) 2018 Sarita Boolchandani et al. This is an open access article distributed under the Creative Commons Attribution License, which permits unrestricted use, distribution, and reproduction in any medium, provided the original work is properly cited.

\begin{abstract}
The indium selenium (InSe) bilayer thin films of various thickness ratios, $\operatorname{In}_{x} \mathrm{Se}_{(1-x)}(x=0.25,0.50,0.75)$, were deposited on a glass substrate keeping overall the same thickness of $2500 \AA$ using thermal evaporation method under high vacuum atmosphere. Electrical, optical, and structural properties of these bilayer thin films have been compared before and after thermal annealing at different temperatures. The structural and morphological characterization was done using XRD and SEM, respectively. The optical bandgap of these thin films has been calculated by Tauc's relation that varies within the range of 1.99 to $2.05 \mathrm{eV}$. A simple low-cost thermoelectrical power measurement setup is designed which can measure the Seebeck coefficient " $S$ " in the vacuum with temperature variation. The setup temperature variation is up to $70^{\circ} \mathrm{C}$. This setup contains a Peltier device TEC1-12715 which is kept between two copper plates that act as a reference metal. Also, in the present work, the thermoelectric power of indium selenide (InSe) and aluminum selenide (AlSe) bilayer thin films prepared and annealed in the same way is calculated. The thermoelectric power has been measured by estimating the Seebeck coefficient for InSe and AlSe bilayer thin films. It was observed that the Seebeck coefficient is negative for InSe and AlSe thin films.
\end{abstract}

\section{Introduction}

Semiconductor compounds formed from group elements II-VI and III-V are extensively used in modern technology [1]. Their bandgap lies between 1.0 and $2.0 \mathrm{eV}$ [2]. They are abundantly available on earth, and they are also both chemically and electrochemically stable in either acid or alkaline condition [3]. Among these materials, selenium is very useful in making optical devices [4-6]. In the present work, InSe bilayer thin films are studied. InSe is used because of its application in optoelectronic devices [7], solar cells [8], and solid-state batteries [9]. The structural, electronic, and electrical properties of InSe were studied by various researchers [10-13].

The thermoelectric (TE) phenomena can provide the direct conversion of applied temperature gradient into electricity (the Seebeck effect) or electricity into temperature difference (the Peltier effect). This solid-state technology can be applied in a variety of applications [14], and the most well known is as a thermocouple for temperature measurement [15]. Nowadays, as the continuous fossil fuel supply decreases and world energy demand increases, TE materials have drawn renewed interest due to their potential to provide a sustainable supply of energy. Compared with other conventional electric generators, the reliability and simplicity of thermoelectricity enable niche applications even though many conventional processes are more efficient.

The Seebeck coefficient $(S)$ measurement as a function of time is one of the significant methods to analyze electronic properties of solids. The $S$ can be used to determine the concentration of majority carrier, type of semiconductor, Fermi level position, and so on [16-19]. In the present work, a setup is designed to calculate the Seebeck coefficient in the vacuum with variations in the temperature from 300 to $310 \mathrm{~K}$. The setup is very compact, low power, and easy to operate. 


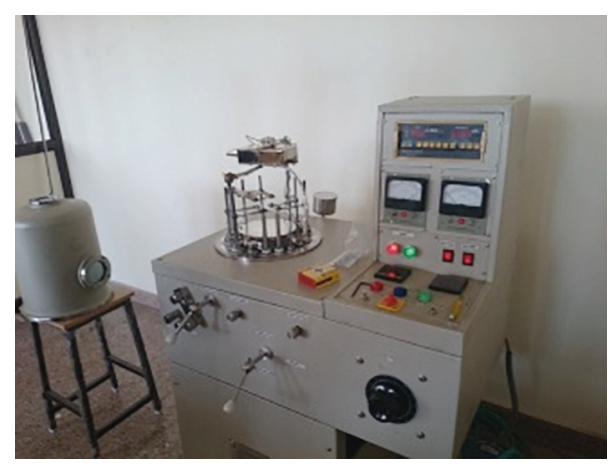

FIgURE 1: Thermal evaporation unit.

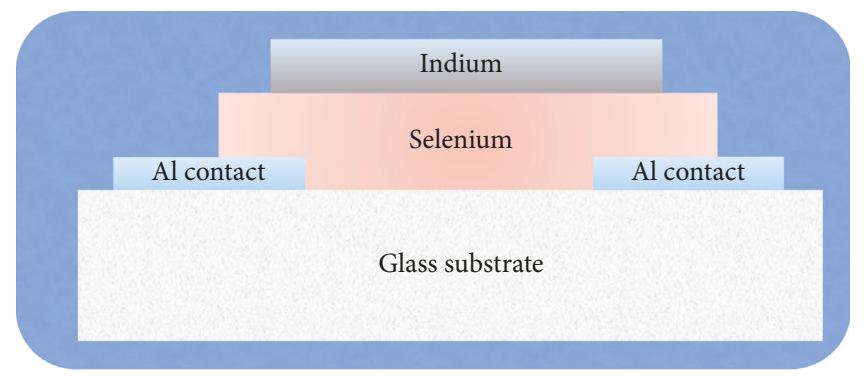

(a)

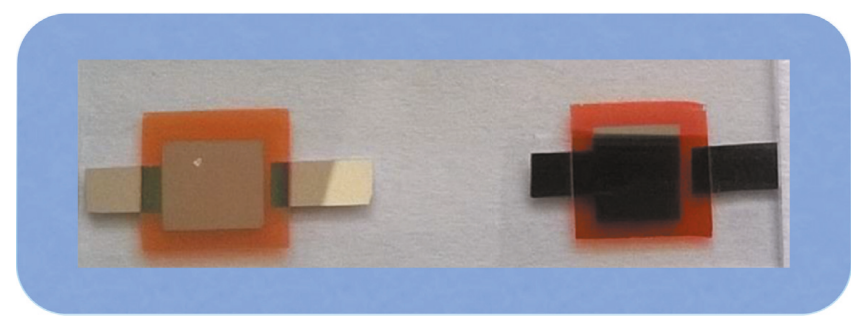

(b)

Figure 2: (a) Schematic representation of InSe thin film structure and (b) prepared thin films.

In this paper, structural, optical, and thermoelectrical properties of InSe bilayer thin films, having different thickness ratios, have been studied.

\section{Experimental}

2.1. Materials and Methods. $\operatorname{In}_{x} \mathrm{Se}_{1-x}$ bilayer thin films of the same thickness $(2500 \AA)$ and different thickness ratios $(x=0.25,0.50$, and 0.75$)$ were deposited by the thermal evaporation method on a clean glass substrate under a vacuum of $10^{-6} \mathrm{mbar}$. The structural, electrical, and optical measurements of the films were carried on to investigate the effect of annealing temperature and thickness ratio. Prepared bilayer films were also annealed at substrate temperatures of $70^{\circ} \mathrm{C}$ and $100^{\circ} \mathrm{C}$. The thin films were prepared using the thermal evaporation coating unit shown in Figure 1.

The prepared bilayered thin film and its schematic diagram are shown in Figure 2.

Thermoelectric power measurement of composite selenium thin films has been carried out in the temperature

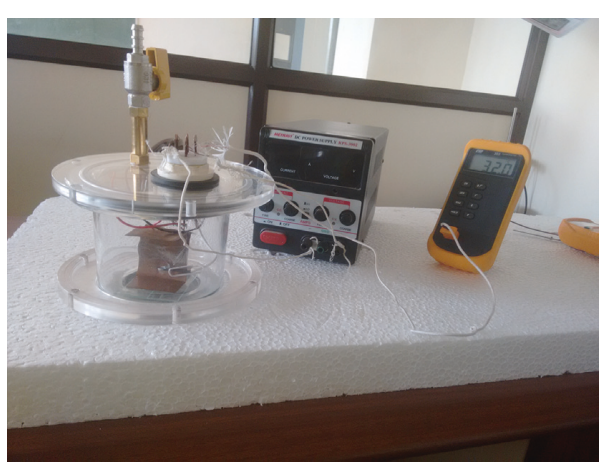

FIgURE 3: Experimental setup for calculation of thermoelectric power.

range of $300-310 \mathrm{~K}$ by taking pure metallic copper $(\mathrm{Cu})$ as a reference metal. The thermoelectric module TEC1-12715 was used for temperature difference in the two-end sample. The module was sandwiched between two copper plates. The experimental setup used for sample testing is shown in Figure 3 . The chamber was pumped first by using a rotary 


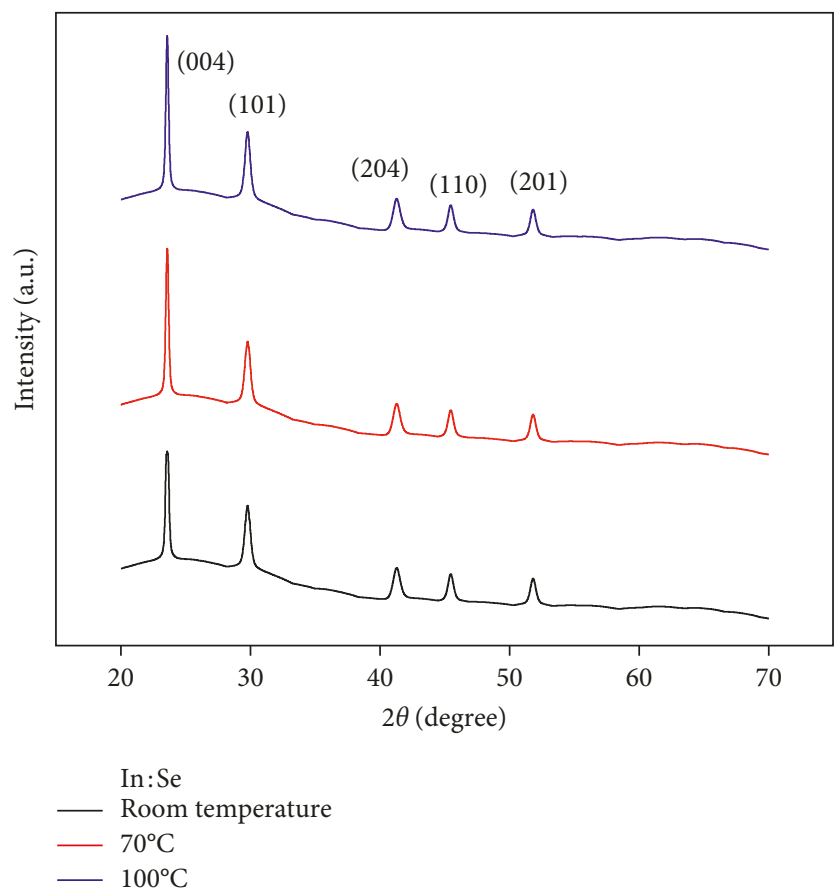

FIgURE 4: XRD patterns of InSe thin films at different annealing temperatures.

pump to a rough vacuum of $10^{-3}$ Torr. Only one end of the sample was heated leading to the creation of a hot junction and a cold junction to create a temperature gradient on the sample.

The thermocouple used for the measurement of the temperature gradient $(d T)$ is of $\mathrm{K}$ type. The sample under investigation was kept on copper plates, and the wires of the module were connected to a constant power supply to provide the voltage to the thermoelectric module in order to maintain the temperature difference. A multimeter was used to measure the voltage difference across the film. The Seebeck coefficient was measured using the following relation:

$$
S=\frac{d V}{d T},
$$

where $d V$ is the voltage difference across the film and $d T$ is the temperature gradient.

\section{Results and Discussions}

3.1. X-Ray Diffraction Study. To identify the structure of the film, the X-ray diffraction method is used. The XRD patterns of the as-deposited and annealed InSe thin film are shown in Figure 4 . The XRD spectrum exhibits the multiple characteristic peaks at $2 \theta=23.5^{\circ}, 29^{\circ}, 41^{\circ}, 45^{\circ}$, and $52.4^{\circ}$ corresponding to the $004,101,204,110$, and 201 phases of indium and selenium. Furthermore, no additional peak was observed for the annealed sample as compared to the asdeposited thin film, indicating that no new interfacial phase was formed after annealing. It has also been observed that only peak intensity is increased with an increase in the annealing temperature, which may be due to the small particle size and ordered distribution of the particles, hence resulting in an increase in the order of the crystalline nature of thin films after heat treatment.

3.2. Scanning Electron Microscopy. The SEM images of the InSe bilayer are shown in Figure 5. Figure 5(a) shows the image before annealing, and Figure 5(b) shows the images after annealing. Figure 5(a) shows that the as-deposited film exhibits a smooth granular structure that appeared uniformly at the surface, and a stack-like structure also appeared due to stack layer deposition. After annealing, the surface morphology of thin films is changed considerably, and it can be seen from Figure 5(b) that big cluster and uneven lumps have appeared in a spherical shape and are distributed on the entire surface. These clusters may be formed due to the agglomeration of particles during heat treatment. According to these SEM images, we may conclude that the as-deposited thin films have partially amorphous structure, while after annealing, the thin films become polycrystalline in nature.

3.3. I-V Characteristics. I-V characteristics of InSe films were estimated using a two-terminal configuration by applying a voltage $(\approx 10 \mathrm{~V})$ to the sample and measuring the current through it using a Keithley 2450 sourcemeter. The measurements were carried out at room temperature, and the variation in logarithm of conductivity with an inverse of temperature for different thickness ratios of InSe thin films with $\pm 2-3 \%$ error is shown in Figure 6 .

It is cleared from figure that conductivity increases with an increase in temperature [20] which indicates the semiconducting behavior of InSe thin films. 


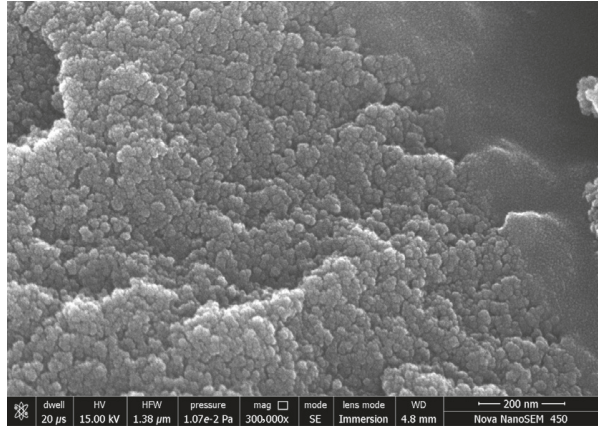

(a)

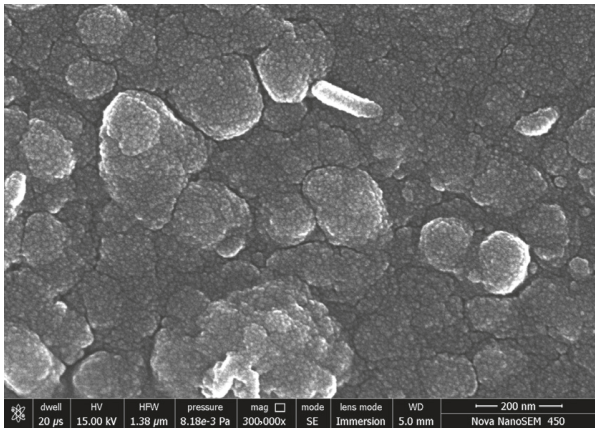

(b)

Figure 5: SEM images of as-deposited (a) and annealed (b) InSe thin films.

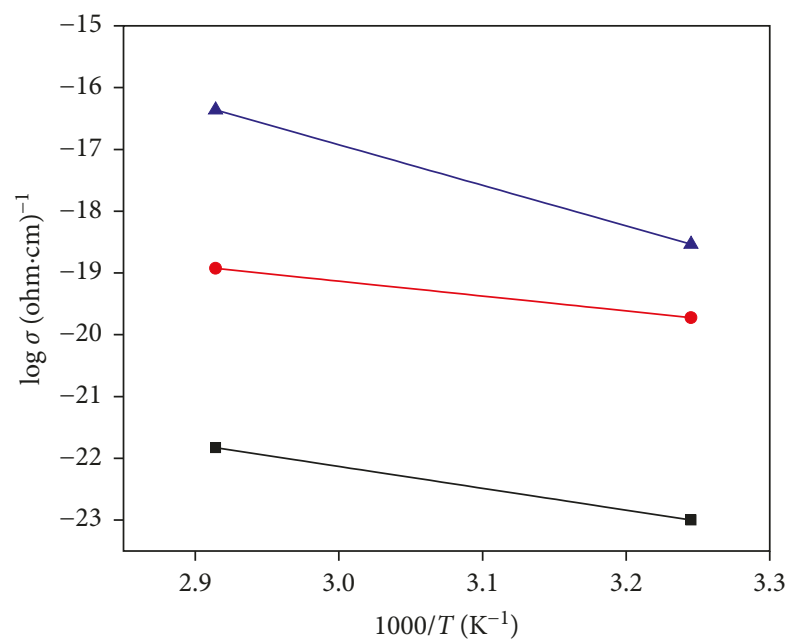

In:Se at room temperature

-m- 25:75

- 50:50

-A- 75:25

Figure 6: Variation in logarithm of electrical conductivity with temperature.

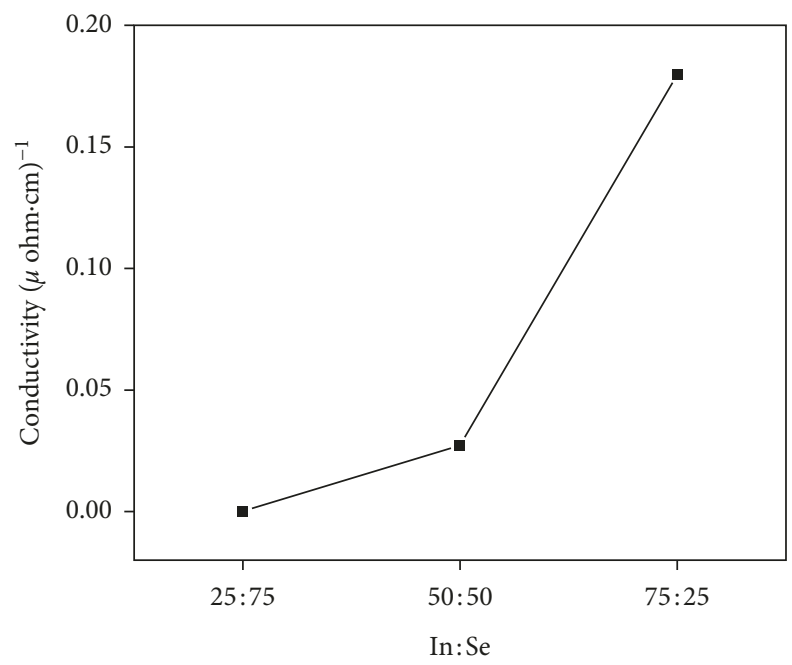

FIgURE 7: Variation in electrical conductivity with In:Se ratio. 

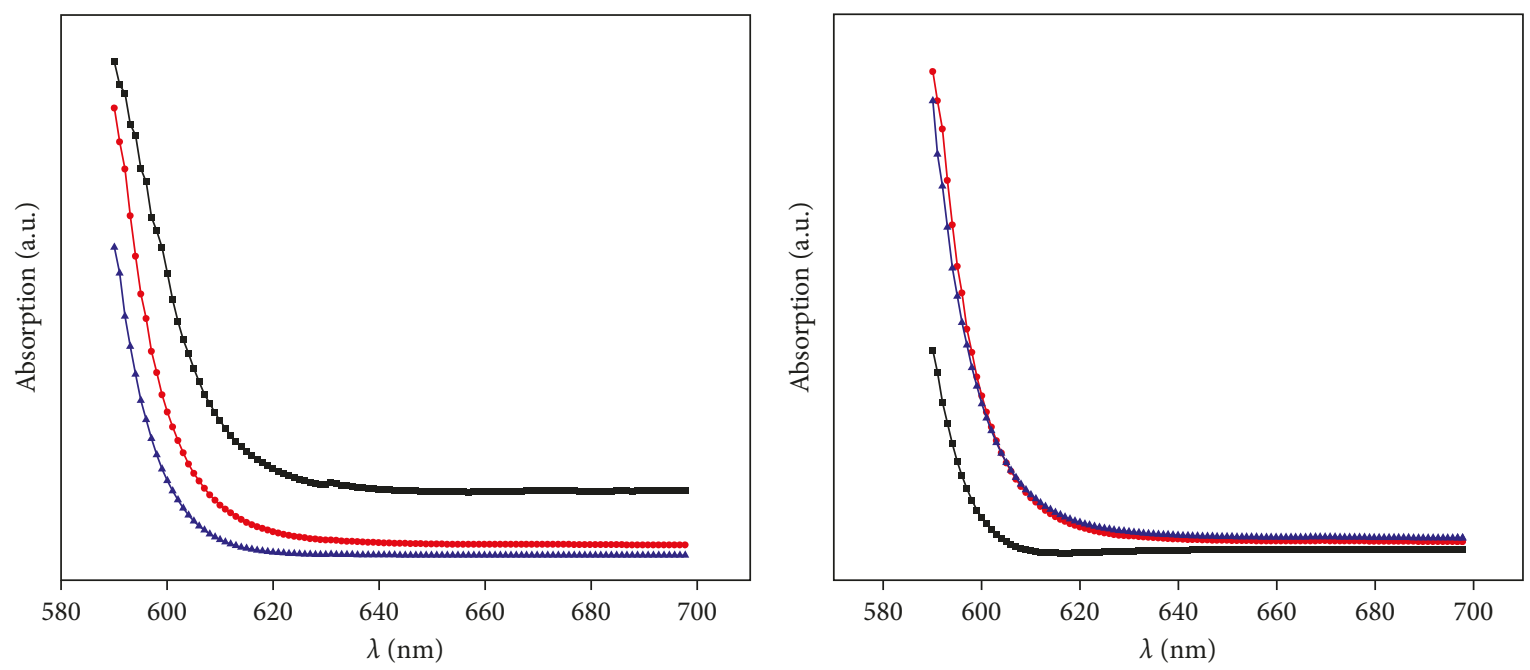

In:Se (50:50)

$\rightarrow$ Room temperature

$\rightarrow 70^{\circ} \mathrm{C}$

In: Se at $70^{\circ} \mathrm{C}$

$\rightarrow$ 25:75

$\rightarrow 50: 50$

$\rightarrow 100^{\circ} \mathrm{C}$

$\longrightarrow 75: 25$

(a)

(b)

FIgURE 8: Optical absorption curves of InSe bilayer thin films for (a) 50:50 thickness ratio at (b) $70^{\circ} \mathrm{C}$.

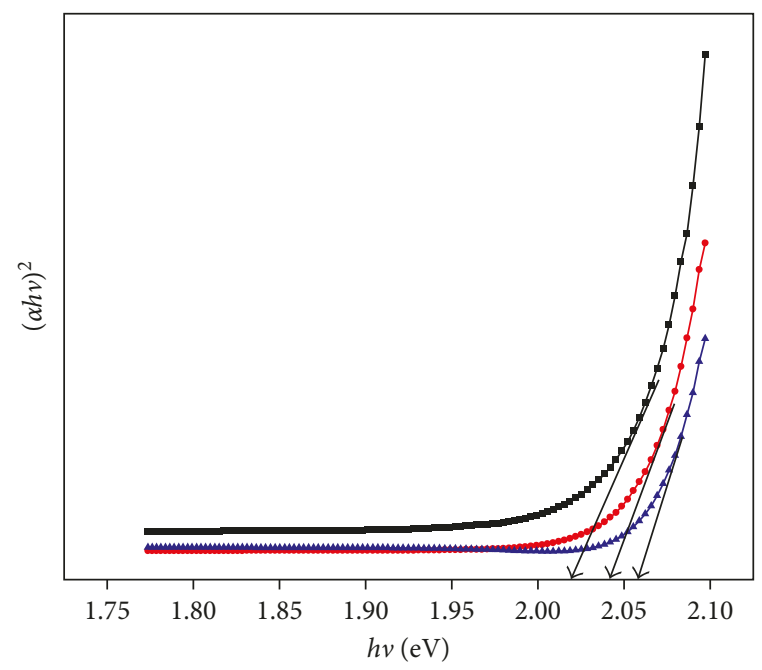

In:Se (25:75)

- Room temperature

$\rightarrow 70^{\circ} \mathrm{C}$

$\leftarrow 100^{\circ} \mathrm{C}$

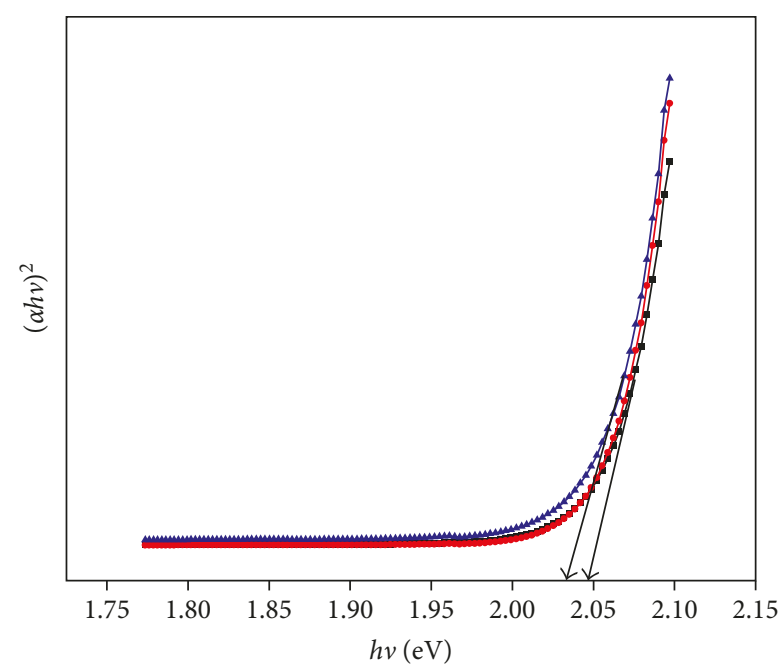

In: Se at $100^{\circ} \mathrm{C}$

$\rightarrow 25: 75$

- 50:50

$\longrightarrow 75: 25$

(a)

(b)

Figure 9: Optical bandgap calculation of InSe bilayer thin films for (a) 25:75 thickness ratio at (b) $100^{\circ} \mathrm{C}$.

An increase in current was observed as the concentration of indium was increased in InSe thin films. This revealed that on increasing the indium concentration in InSe bilayer thin films, the room temperature film conductivity is increased from $2.13 \times 10^{-10}$ to $1.80 \times 10^{-7}(\sigma-\mathrm{cm})^{-1}$ in comparison with that of lower concentration of indium, as shown in Figure 7. It may be attributed to the interaction of metallic In with the Se thin film layer, which leads to a decrease in the potential difference at the interface of the InSe bilayer thin film, which in turn is expected to form the crystalline structure of InSe bilayer thin films; hence, the electrical resistance of composite films decreases or the conductivity increases. 
TABLE 1: Bandgap variation of InSe with different thickness ratios.

\begin{tabular}{lccc}
\hline \multirow{2}{*}{ Thickness ratio } & & Bandgap $(\mathrm{eV})$ & \\
& $\mathrm{RT}$ & $70^{\circ} \mathrm{C}$ & $100^{\circ} \mathrm{C}$ \\
\hline $25: 75$ & 2.01 & 2.04 & 2.05 \\
$50: 50$ & 1.99 & 2.02 & 2.04 \\
$75: 25$ & 2.01 & 2.03 & 2.04 \\
\hline
\end{tabular}

TABLE 2: Bandgap variation of InSe with different annealing temperatures.

\begin{tabular}{lccc}
\hline Temperature $\left({ }^{\circ} \mathrm{C}\right)$ & & Bandgap $(\mathrm{eV})$ & $75: 25$ \\
\hline RT & $25: 75$ & $50: 50$ & 2.03 \\
70 & 2.02 & 2.01 & 2.02 \\
100 & 2.05 & 2.02 & 2.03 \\
\hline
\end{tabular}

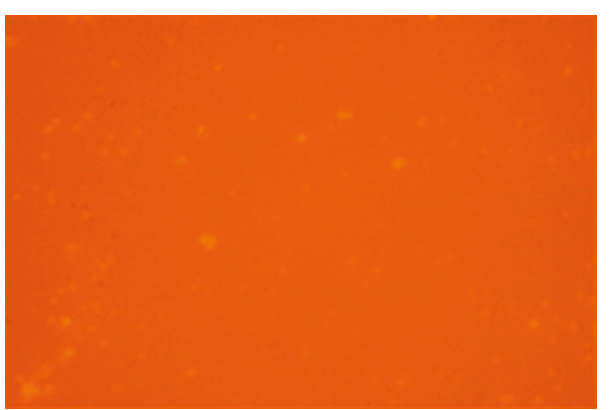

(a)

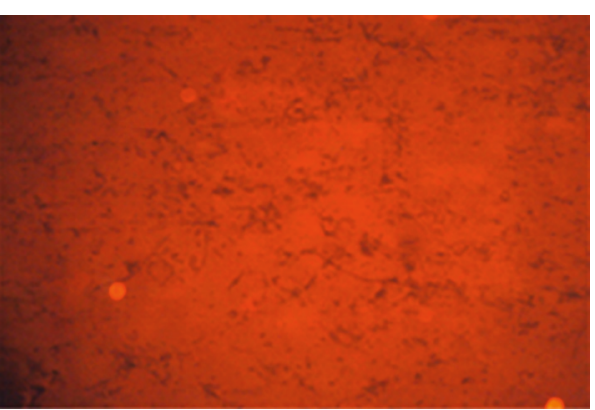

(b)

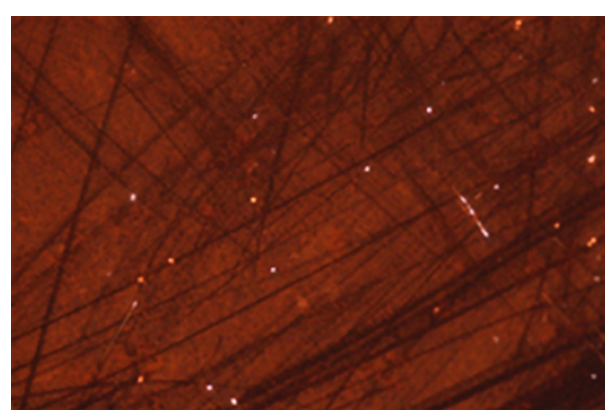

(c)

Figure 10: Optical micrographs of InSe thin films with different thickness ratios: (a) 25:75, (b) 50:50, and (c) 75:25.

3.4. Optical Properties. In the high absorption region, absorption coefficient $(\alpha)$ can be represented according to Tauc [21] and Pankove [22], respectively, as

$$
\alpha h v=A(h v-E g)^{n},
$$

where $A$ is a constant depending on the transition probability, $E g$ is the optical bandgap, and $n$ is the index that characterizes the optical absorption process and is theoretically equal to $2,1 / 2,3$, and $3 / 2$ for indirect allowed [23], direct allowed, indirect forbidden, and direct forbidden transition, respectively. The method for determining the value of bandgap $E g$ is obtained from the graph of $(\alpha h \nu)^{1 / n}$ versus photon energy $h \nu$. If an appropriate value of $n$ is used to obtain a linear plot, the value of $E g$ will be given by the intercept on the $h v$ axis.

The absorption edge of the InSe bilayer thin film is located in a visible range, that is, from $580 \mathrm{~nm}$ to $620 \mathrm{~nm}$, as shown in Figure 8. It was observed that at a low concentration of indium absorption is low as the annealing temperature increases, while for high indium concentration, absorption increases with an increase in the annealing temperature that may be due to an increase in energy density because of higher concentration of indium which was further increased with the annealing temperature. 

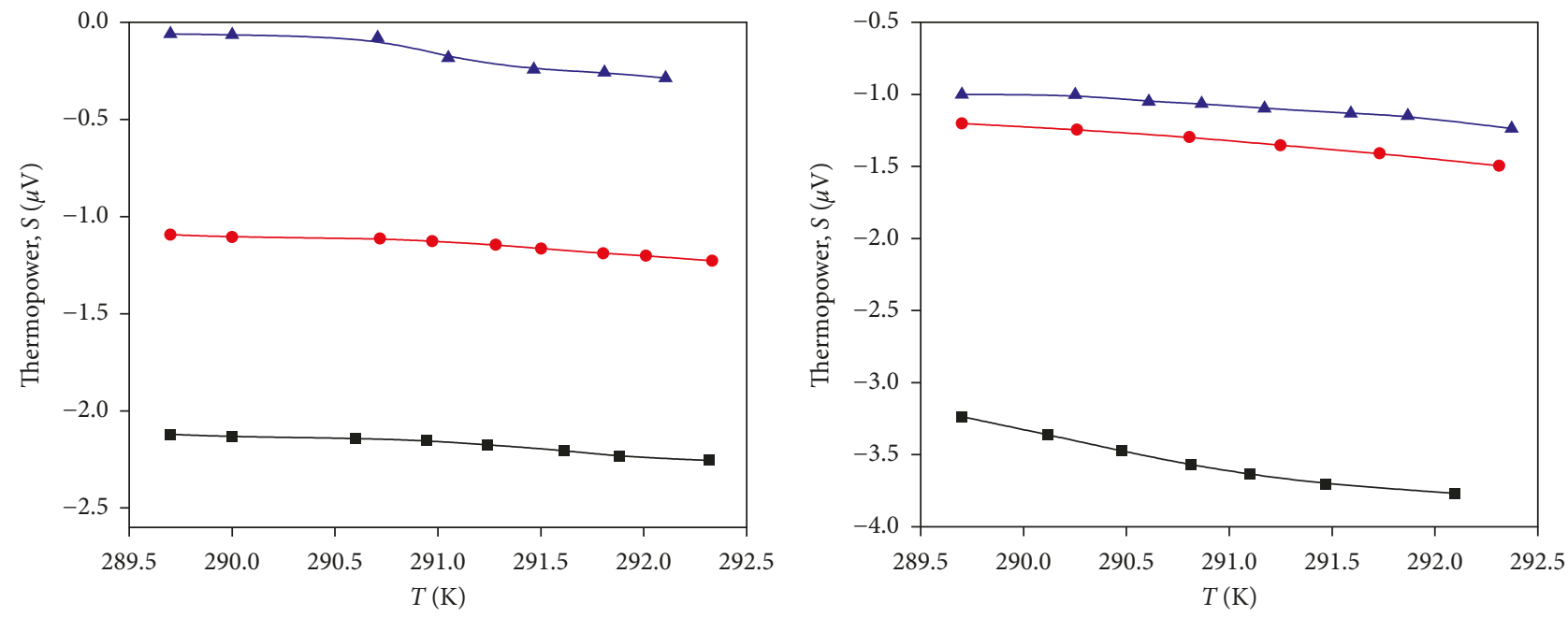

$$
\begin{aligned}
& \text { In:Se at RT } \\
& -\quad 25: 75 \\
& -\quad 50: 50 \\
& \simeq 75: 25
\end{aligned}
$$

$$
\begin{aligned}
& \text { In:Se at } 100^{\circ} \mathrm{C} \\
& -25: 75 \\
& --50: 50 \\
& \leftarrow 75: 25
\end{aligned}
$$

(a)

(b)

Figure 11: Graph between thermoelectric power and temperature for InSe: (a) RT and (b) $100^{\circ} \mathrm{C}$.
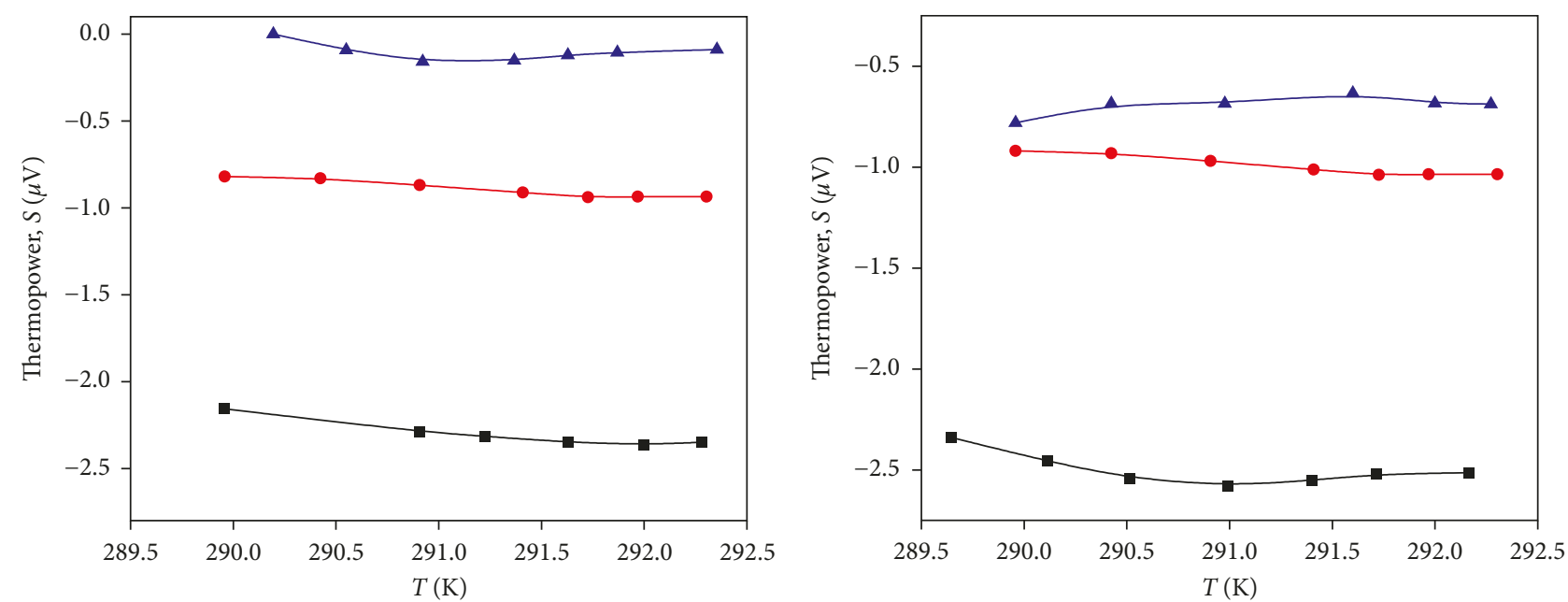

$$
\begin{aligned}
& \mathrm{Al}: \text { Se at RT } \\
& -\quad 25: 75 \\
& \longrightarrow-50: 50 \\
& \simeq 75: 25
\end{aligned}
$$

$$
\begin{aligned}
& \mathrm{Al}: \text { Se at } 100^{\circ} \mathrm{C} \\
& -\quad 25: 75 \\
& \rightarrow-50: 50 \\
& \simeq 75: 25
\end{aligned}
$$

(a)

(b)

Figure 12: Graph between thermoelectric power and temperature for AlSe: (a) RT and (b) $100^{\circ} \mathrm{C}$.

The bandgap of the prepared InSe bilayer thin film was calculated using Tauc's relation and is shown in Figure 9. The bandgap value is summarized in Tables 1 and 2 .

3.5. Optical Micrographs. Optical micrographs of the InSe bilayer were taken with the help of a USB optical microscope and are shown in Figure 10, which shows that the InSe bilayer thin film surface is smooth and no cracks are there.
3.6. Thermoelectric Power. The thermoelectromotive force, that is, the Seebeck coefficient of the prepared InSe and AlSe bilayer thin films with different thickness ratios and annealing temperatures is shown in Figures 11-14.

As shown in Figures 11 and 12, the increase of conductivity with a decreasing Se ratio in composition indicates the semimetallic behavior.

Generally, the Seebeck coefficients increase with the increase in annealing temperature as shown in Figures 13 

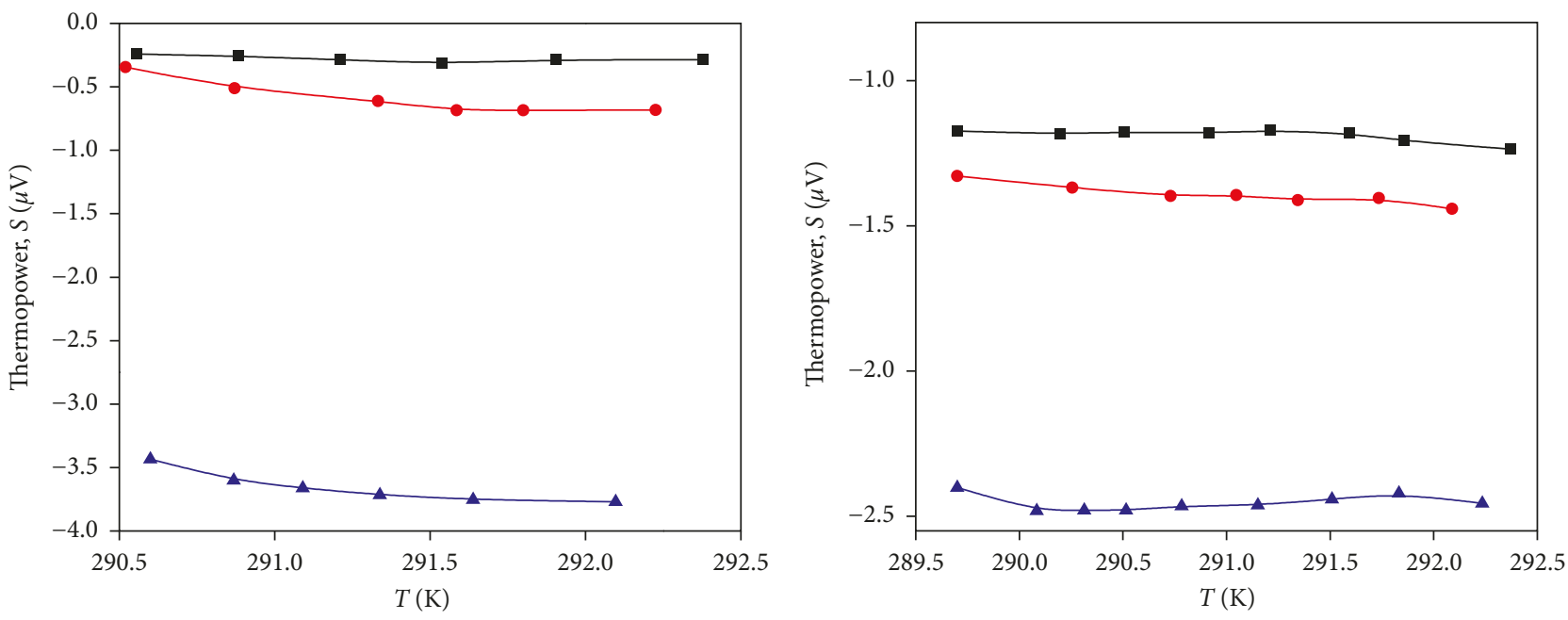

$$
\begin{aligned}
& 25: 75 \mathrm{In}: \mathrm{Se} \\
& -\mathrm{RT} \\
& \rightarrow-70^{\circ} \mathrm{C} \\
& \leftarrow 100^{\circ} \mathrm{C}
\end{aligned}
$$

$$
\begin{aligned}
& 75: 25 \mathrm{In}: \mathrm{Se} \\
& -\mathrm{RT} \\
& -\quad 70^{\circ} \mathrm{C} \\
& \leftarrow \quad 100^{\circ} \mathrm{C}
\end{aligned}
$$

(a)

(b)

FIGURE 13: Graph between thermoelectric power and temperature for InSe: (a) 25:75 and (b) 75:25.

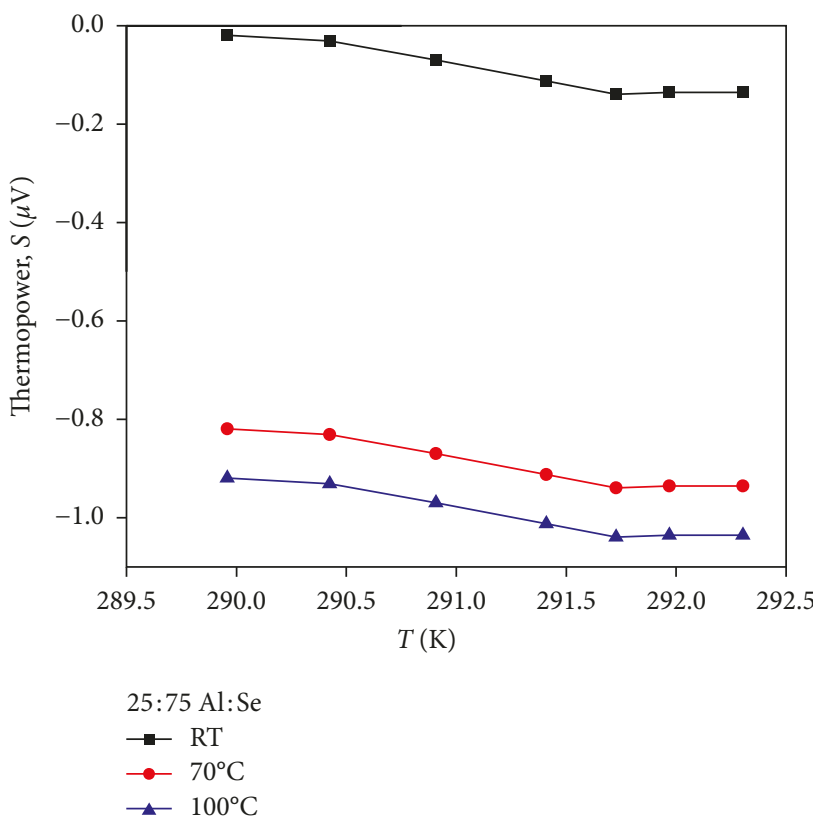

(a)

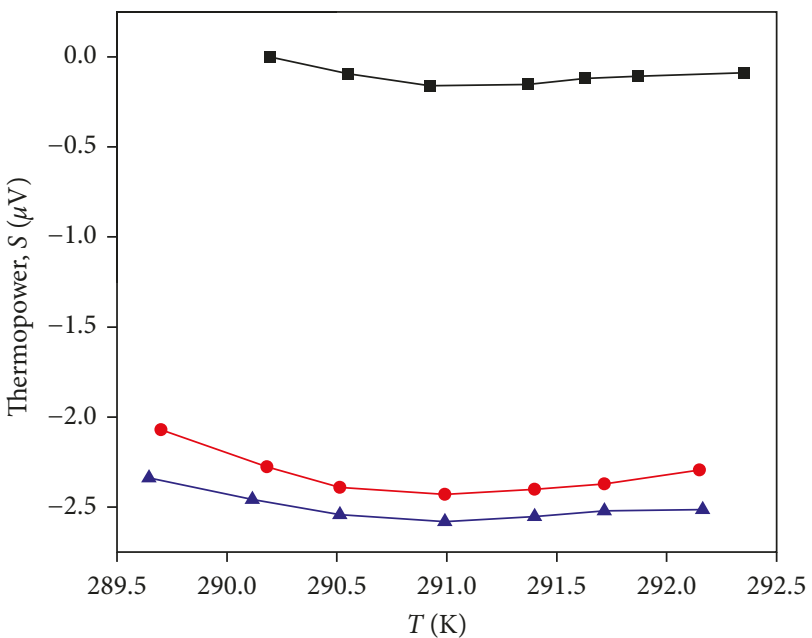

$$
\begin{aligned}
& 75: 25 \mathrm{Al}: \mathrm{Se} \\
& -\mathrm{RT} \\
& \rightarrow-70^{\circ} \mathrm{C} \\
& -100^{\circ} \mathrm{C}
\end{aligned}
$$

(b)

Figure 14: Graph between thermoelectric power and temperature for AlSe: (a) 25:75 and (b) 75:25.

and 14. This may be due to the better crystallinity and elimination of grain boundaries of the thin films at higher annealing temperatures.

The Seebeck coefficient of the deposited films has found to be negative, that is, electrons are predominant charge carriers which confirm the n-type behavior of indium selenide and aluminum selenide thin films [24].

\section{Conclusion}

InSe bilayer thin films with different thickness ratios have been prepared by the vacuum evaporation technique. The films were uniform and had good adherence to the substrate. The XRD of InSe films confirms the formation of InSe. SEM images show that the as-deposited films are smooth, while 
after annealing, the thin films become polycrystalline in nature. The bandgap energies of InSe thin films vary with the variation in thickness ratio and annealing temperature. The increase in conductivity with an increase in the temperature confirms the semiconducting behavior of the InSe films. Also, conductivity increases with an increase in the In : Se ratio in the films. The negative Seebeck coefficient confirms the n-type behavior of the deposited InSe and AlSe thin films.

\section{Conflicts of Interest}

The authors declare that they have no conflicts of interest.

\section{Acknowledgments}

The authors thank Material Research Laboratory, Vivekananda Global University, Jaipur; MRC, MNIT, Jaipur, for XRD and SEM characterization; and Dr. S. S. Sharma Ajmer for providing facilities for characterization.

\section{References}

[1] M. A. Majeed khan, M. Zulfequar, and M. Husain, "Estimation of density of localized states of a-Se100-xSbx films using electrical properties," Journal of Physics and Chemistry of Solids, vol. 62, no. 6, pp. 1093-1101, 2001.

[2] H. Zishan, M. M. Khani, M. M. Zulfequar, and M. Husain, "Electrical conduction mechanism in a-Se80-xTexGa20films," Journal of Physics: Condensed Matter, vol. 7, no. 47, pp. 8979-8991, 1995.

[3] R. M. Mehra, H. Kumar, S. Koul, and P. C. Mathur, "Thickness dependence of DC conductivity of amorphous Se and binary amorphous SeTe, SeGe, and SeSb films," Physica Status Solidi (a), vol. 83, no. 1, p. 341, 1984.

[4] S. Kumar, R. Arora, and A. Kumar, "High-field conduction in a-Se80Te20 and a-Se80Te10M10 (M=Ag, Cd or Sb)," Physica B: Condensed Matter, vol. 183, no. 1-2, pp. 172-178, 1993.

[5] M. M. Hafiz, A. H. Moharram, M. A. Abdel-Rehim, and A. A. Abu-Sehly, "The effect of annealing on the optical absorption and electrical conduction of amorphous As24.5Te71Cd4.5 thin films," Thin Solid Films, vol. 292, no. 7, 1997.

[6] Z. H. Khan, M. Zulfequar, and M. J. Husain, "Optical properties of a-Se80-xTexGa20thin films," Journal of Optics, vol. 28, no. 4, pp. 151-157, 1997.

[7] K. Abe, H. Takebe, and K. Maronaga, "Preparation and properties of GeGaS glasses for laser hosts," Journal of NonCrystalline Solids, vol. 212, no. 2-3, pp. 143-150, 1997.

[8] K. Wei, D. P. Machewirth, J. Wenzel, and G. H. Sigel, "Pr3+-doped GeGaS glasses for $1.3 \mu \mathrm{m}$ optical fiber amplifiers," Journal of Non-Crystalline Solids, vol. 182, no. 2, pp. 257-261, 1995.

[9] K. Tanaka, "Structural phase transitions in chalcogenide glasses," Physical Review B, vol. 39, no. 2, pp. 1270-1279, 1989.

[10] B. Kobbi and N. Kesri, "Physico-chemical and electrical properties of InSe films," Vacuum, vol. 75, no. 2, pp. 177-182, 2004.

[11] A. Hirohata, J. S. Moodera, and G. P. Berera, "Structural and electrical properties of InSe polycrystalline films and diode fabrication," Thin Solid Films, vol. 510, no. 1-2, pp. 247-250, 2006.

[12] F. I. Mustafa, S. Gupta, N. Goyal, and S. K. Tripathi, "Effect of indium concentration on the electrical properties of InSe alloy," Physica B: Condensed Matter, vol. 405, no. 19, pp. 4087-4091, 2010.

[13] P. Matheswaran, R. Saravana Kumar, and R. Sathyamoorthy, "Effect of annealing on the structural and optical properties of InSe bilayer thin films," Vacuum, vol. 85, no. 8, pp. 820-826, 2011.

[14] S. B. Riffat and X. Ma, "Thermoelectrics: a review of present and potential applications," Applied Thermal Engineering, vol. 23, no. 8, pp. 913-935, 2003.

[15] P. A. Kinzie, Thermocouple Temperature Measurement, Wiley, New York, NY, USA, 1973.

[16] K. C. Kao and W. Hwang, Electrical Transport in Solids, Vol. 14, Pergamon Press, Oxford, UK, 1981.

[17] M. Cutler, "The thermoelectric behaviour of disordered systems," Philosophical Magazine, vol. 25, no. 1, p. 173, 1972.

[18] M. Cutler and N. F. Mott, "Observation \& Anderson localization in an electron gas," Physical Review, vol. 181, no. 3, pp. 1336-1340, 1969.

[19] V. A. Johnson, "Seebeck effect in semiconductors," in Progress in Semiconductors, Vol. 1, Heywood, London, UK, 1956.

[20] C. Viswanathan, G. G. Rusu, S. Gopal, D. Mangalaraj, and S. K. Narayandass, "On the electrical characteristics of vacuum evaporated indium selenide thin films," Journal of $O p$ toelectronics and Advanced Materials, vol. 7, pp. 705-711, 2005.

[21] J. Tauc, Amorphous and Liquid Semiconductors, Chapter 4, Plenum Press, New York, NY, USA, 1974.

[22] J. I. Pankove, Optical Processes in Semiconductors, PrenticeHall, New Jersey, NY, USA, 1971.

[23] A. V. Savitskii, M. V. Kurik, and K. D. Tovstyuk, "Optical absorption in Zn, HgI, Te thin films," Optics and Spectroscopy, vol. 19, pp. 56-73, 1964.

[24] J. Hossain, M. Julkarnain, K. Shaifullah Sharif, and K. A. Khan, "Preparation and properties of indium selenide (InSe) thin films for selective surface applications," Journal of Physical Science and Application, vol. 1, pp. 37-43, 2011. 


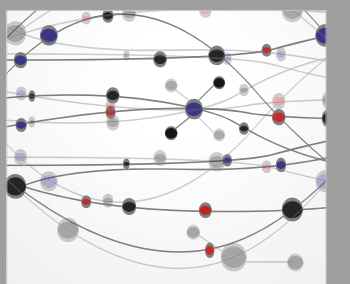

The Scientific World Journal
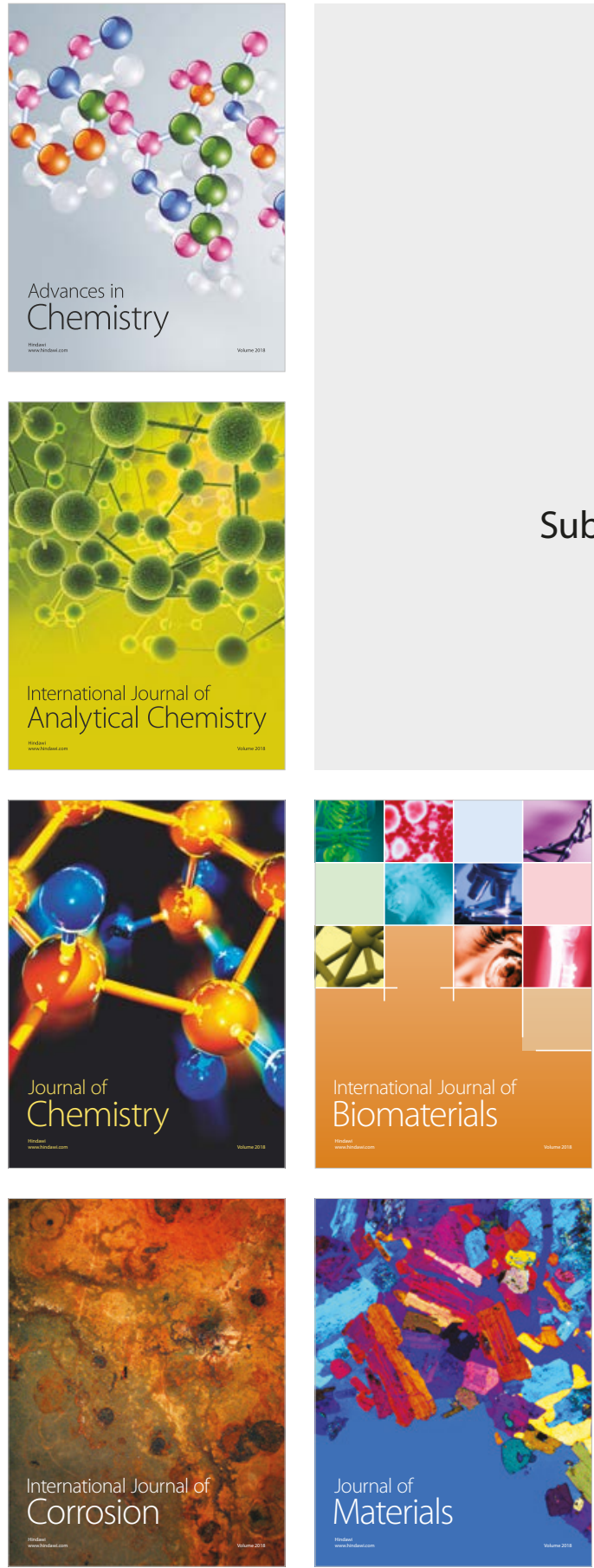

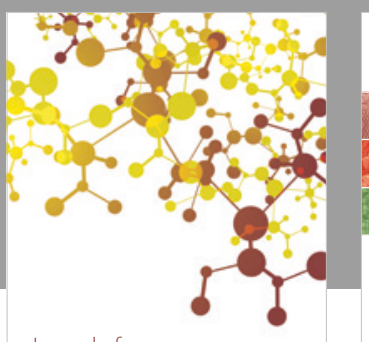

Journal of

Applied Chemistry
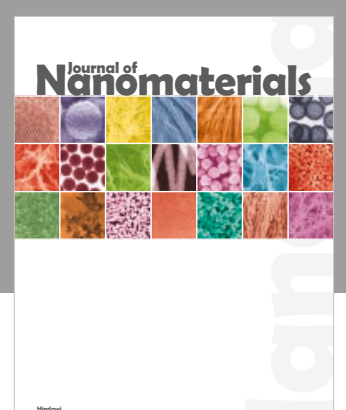

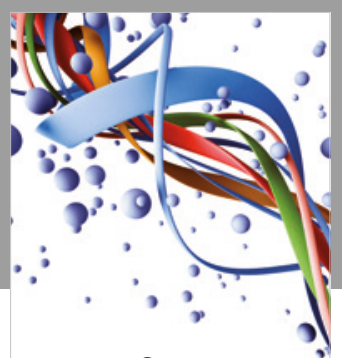

Scientifica

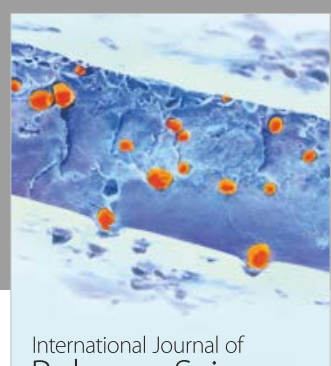

Polymer Science

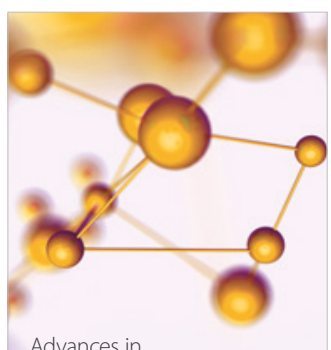

Physical Chemistry
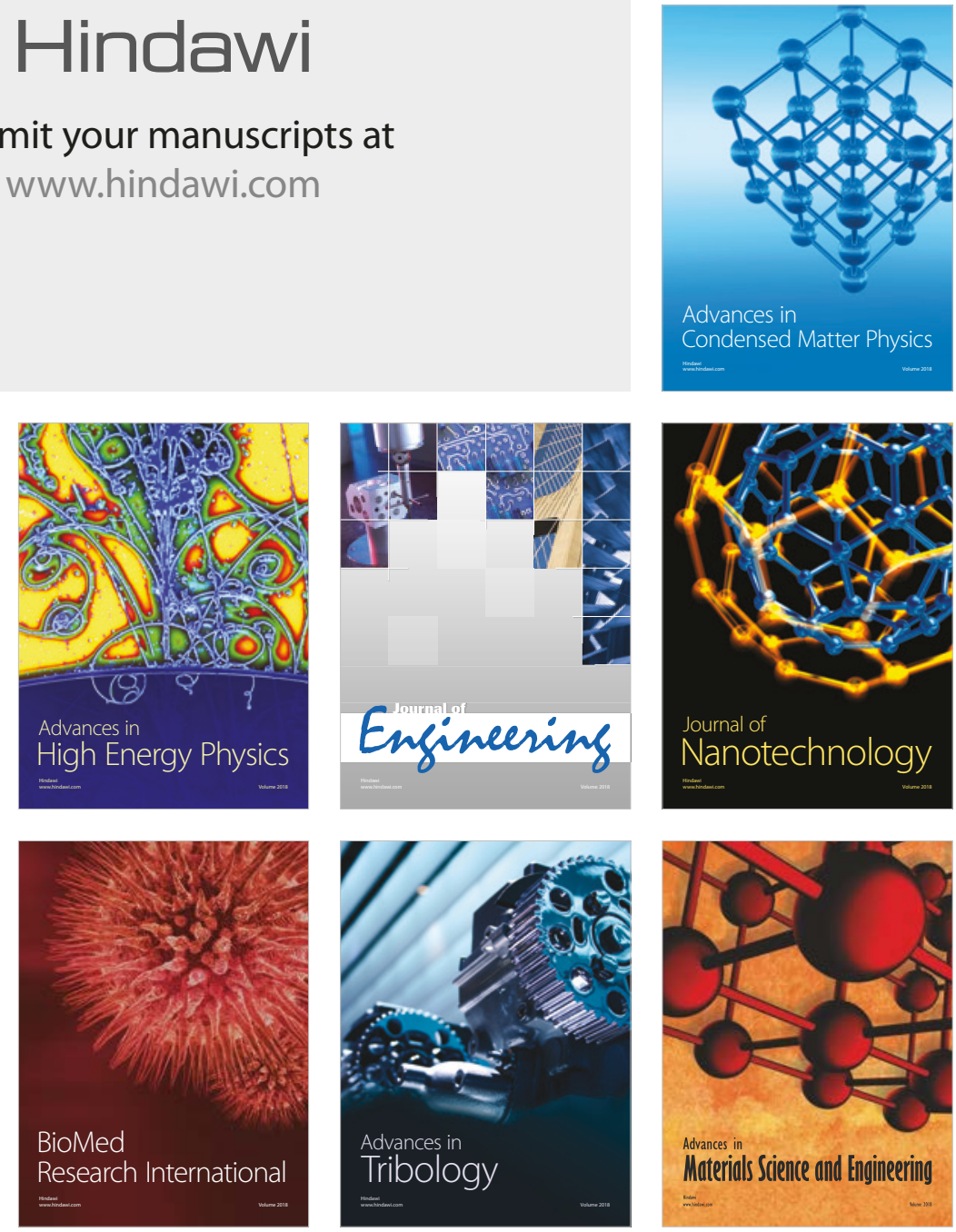